\title{
AVALIAÇÃO NUMÉRICA DA INFLUÊNCIA DO PARÂMETRO ESPESSURA NO DESEMPENHO DAS TEORIAS FSDT E HSDT APLICADAS A PLACAS COMPÓSITAS*
}

Cassiele Renata da Silva ${ }^{1}$

Resumo

Adriana Amaro Diacenco ${ }^{2}$

O presente trabalho trata-se da modelagem por elementos finitos de materiais compósitos reforçados com fibras de carbono utilizando duas teorias: a Teoria da Deformação Cisalhante de Primeira Ordem (FSDT) e a Teoria da Deformação Cisalhante de Ordem Superior (HSDT), com o objetivo de efetuar um comparativo entre ambas no contexto de sua capacidade de predição do comportamento dinâmico, sendo possível avaliar e ilustrar as características de desempenho de ambas as formulações. Além disso, analisando o parâmetro geométrico da espessura das placas compósitas torna-se possível comprovar a limitação de uma das teorias. Os resultados são apresentados na forma de funções de resposta em frequência (FRF's) onde é avaliada computacionalmente a presença de ruídos nos resultados.

Palavras-chave: Material compósito; FSDT; HSDT; Espessura.

\section{NUMERICAL EVALUATION OF THE INFLUENCE OF THE THICKNESS PARAMETER IN THE FSDT AND HSDT THEORIES APPLIED TO COMPOSITE PLATE MATERIALS}

\begin{abstract}
This paper is about the Finite Element Modeling of composite materials reinforced with carbon fiber, using two theories: First-order Shear Deformation Theory (FSDT) and Higher-order Shear Deformation Theory (HSDT). The Purpose of this paper is making a comparison between these theories in the context of its dynamic prediction ability, being possible to evaluate and illustrate the characteristics and the performance in both situations. Moreover, analyzing the geometric parameter of thickness of the composite plates it becomes possible to prove the limitation of one of the theories. The results are presented as frequency response functions (FRF's) which are evaluated computationally for the presence of noise in the results.
\end{abstract}

Keywords: Composite material; FSDT; HSDT; Thickness.

\footnotetext{
${ }^{1}$ Graduanda Engenharia de Produção, Centro Universitário de Itajubá - fepi, Itajubá, Brasil.

2 Professora e pesquisadora do Centro Universitário de Itajubá - fepi, Doutora em Engenharia Mecânica, Itajubá, Brasil.
} 


\section{INTRODUÇÃO}

Materiais compósitos são materiais que vêm sendo amplamente aplicados em diversos setores industriais variando desde a indústria aeronáutica a indústria petrolífera. Com este crescimento, torna-se significativo conhecer o comportamento mecânico e caracterizar estes materiais numericamente, uma vez que os modelos numéricos, em muitas situações, podem ser mais acessíveis do que experimentos em escala real, sendo mais vantajoso representar e entender o comportamento destes materiais por simulações numéricas para depois realizar experimentos.

As limitações apresentadas pelos modelos analíticos, cuja aplicabilidade fica restrita a problemas simples e para a sua aplicação geralmente exigem hipóteses de difícil ocorrência na prática, tais como homogeneidade das características do material, isotropia e linearidade de resposta, nos últimos anos um método que vem sendo amplamente aplicado é o método de elementos finitos.

O Método de Elementos Finitos surgiu na década de 1950, devido à necessidade de projetar estruturas de modelos contínuos. Os primeiros elementos formam concebidos por engenheiros aeronáuticos para a análise de distribuição de tensões em chapas de asa de avião (Soriano [1]).

$\mathrm{Na}$ técnica por elementos finitos, o campo de deslocamentos mecânicos de um determinado modelo é aproximado através de uma série polinomial ao longo da espessura. Existem diversas teorias utilizadas por elementos finitos que são classificadas de acordo com esta série polinomial, as que são mais utilizadas são: a Teoria da Deformação Cisalhante de Primeira Ordem (FSDT) e a Teoria da Deformação Cisalhante de Ordem Superior (HSDT) (Reddy [2]). A escolha de qual destas teorias utilizarem dependerá do tipo de problema, por exemplo, a Teoria FSDT consegue descrever o comportamento mecânico de placas finas e moderadamente espessas, nesse sentido este trabalho apresenta uma avaliação numérica comparativa entre a Teoria FSDT e HSDT quando o parâmetro geométrico espessura da camada compósita varia (CEN et al. [3]). Enquanto que, a Teoria HSDT é a que mais se aproxima dos resultados obtidos pela teoria da elasticidade, porém é uma teoria que apresenta um elevado número de graus de liberdade se comparado a Teoria FSDT.

As respostas dinâmicas e a avaliação da confiabilidade de sistemas estruturais, como placas constituídas de material compósito, podem ser muito sensíveis a certas variações, tais como: geometria, propriedades mecânicas e condições de contorno e, também ao tipo de modelo matemático utilizado para descrever o comportamento mecânico destes materiais.

Na modelagem dos materiais compósitos os parâmetros de projetos mais relevantes no estudo de compostas são: a orientação das fibras reforçadas e a espessura das camadas.

Baseado no que foi exposto acima este trabalho apresenta a modelagem de materiais compósitos do tipo laminado por meio das teorias FSDT e HSDT e avalia os resultados através das funções de resposta em frequência (FRF).

\section{FORMULAÇÃO NUMÉRICA PELA TEORIA FSDT}

O campo de deslocamentos mecânicos representado pela Teoria da Deformação Cisalhante de Primeira Ordem é expresso por:

$$
\boldsymbol{U}(x, y, z, t)=\boldsymbol{A}(z) \boldsymbol{u}(x, y, t)
$$


Na Equação (1):

$$
\begin{aligned}
& \boldsymbol{U}(x, y, z, t)=[u(x, y, z, t) v(x, y, z, t) w(x, y, z, t)]^{T} \\
& A(z)=\left[\begin{array}{lllll}
1 & 0 & 0 & z & 0 \\
0 & 1 & 0 & 0 & z \\
0 & 0 & 1 & 0 & 0
\end{array}\right] \\
& \boldsymbol{u}(x, y, t)=\left[u_{0}(x, y, t) v_{0}(x, y, t) w_{0}(x, y, t) \psi_{x}(x, y, t) \psi_{y}(x, y, t)\right]^{T}
\end{aligned}
$$

Onde: $\Psi_{x}$ e $\Psi_{y}$ são as rotações dos segmentos normais à superfície de referência em torno dos eixos y e $\mathrm{x}$ respectivamente.

As variáveis mecânicas apresentadas na Eq. (1) são convertidas para sua formulação em elementos finitos usando funções de forma apropriadas. O elemento considerado, ilustrado na figura 1, é um elemento retangular de placa que apresenta três nós por aresta, perfazendo um total de oito nós, sendo conhecido como elemento Serendipity (REDDY [1]).

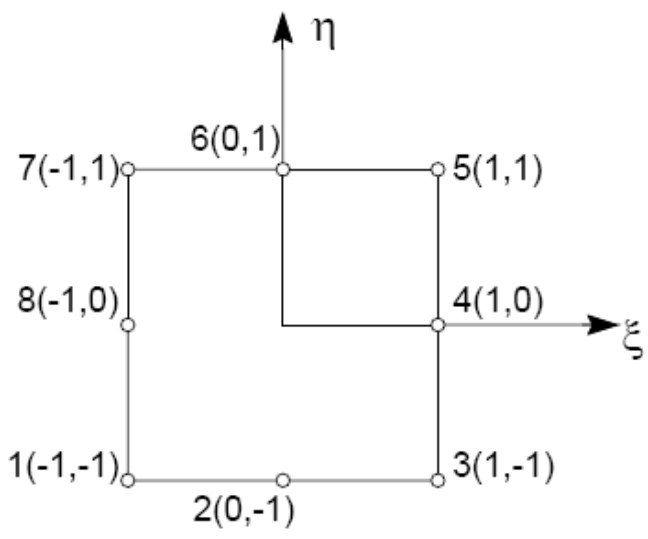

(A)

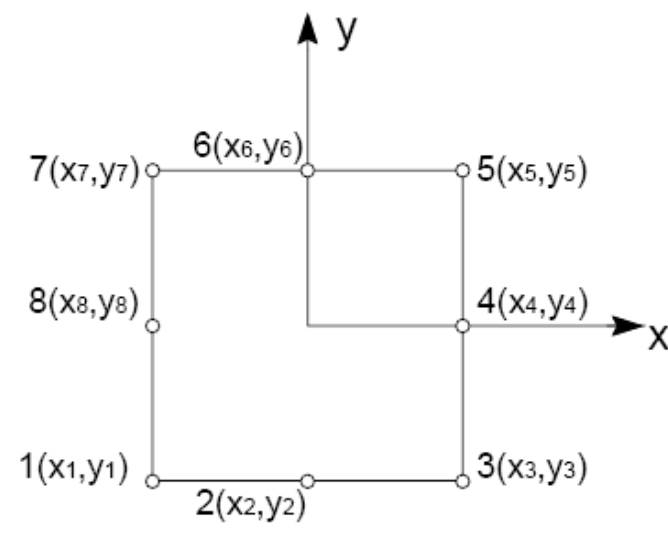

(B)

Figura 1 - Forma retangular do elemento empregado na formulação por elementos finitos (a) coordenadas locais (b) coordenadas globais.

As funções de forma, que relaciona os deslocamentos em um ponto qualquer com os deslocamentos nos pontos nodais, da família Serendipity são apresentadas na Eq. (2).

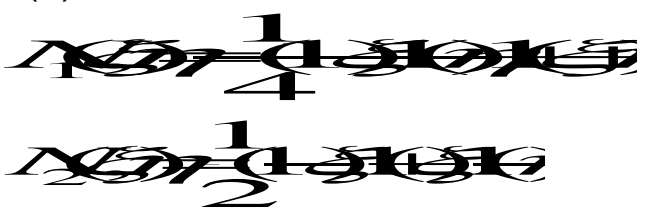




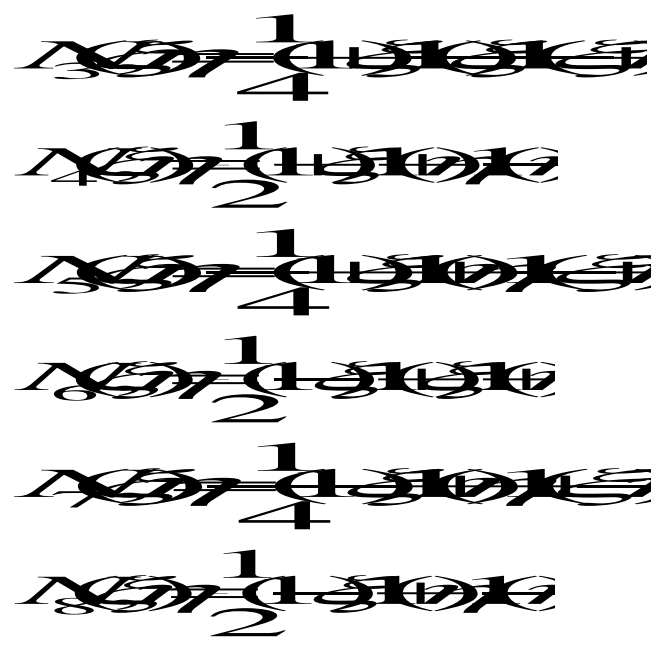

A expressão eq. (1) pode ser escrita em termos das funções de forma como:

$$
\{\hat{u}(\xi, \eta, t)\}_{(5 \times 1)}=\left[N_{u}(\xi, \eta, t)\right]_{5 \times 40}\left\{u_{e}(t)\right\}_{40 \times 1}
$$

Da Teoria da Elasticidade Linear tem-se que as deformações mecânicas são definidas em termos das formas diferenciais dos deslocamentos mecânicos (Reddy [1]) expressos na Eq. (4):

$$
\begin{aligned}
& \varepsilon_{x x}=\frac{\partial u}{\partial x}, \varepsilon_{y y}=\frac{\partial v}{\partial y}, \varepsilon_{z z}=\frac{\partial w}{\partial z} \\
& \gamma_{x y}=\frac{\partial u}{\partial y}+\frac{\partial}{\partial x}, \gamma_{x z}=\frac{\partial u}{\partial z}+\frac{\partial u}{\partial y}, \gamma_{y z}=\frac{\partial}{\partial z}+\frac{\partial u}{\partial}
\end{aligned}
$$

Utilizando as relações expressão na Eq (4) e separando os efeitos em efeito de flexão-membrana e cisalhamento obtém-se:

$$
\begin{aligned}
& \varepsilon_{b}(x, y, z, t)=\left[D_{0}+z D_{1}\right] u(x, y, t)=D_{b}(z) u(x, y, t) \\
& \varepsilon_{s}(x, y, z, t)=\left[D_{4}\right] u(x, y, t)=D_{s}(z) u(x, y, t)
\end{aligned}
$$

As matrizes $D_{i}(i=0, \ldots, 6)$ são formadas por operadores diferenciais.

Podemos expressar as deformações de flexão $\varepsilon_{b}$ e deformações de cisalhamento transversais $\varepsilon_{s}$ em coordenadas locais, combinando as relações (6) com a Eq.(5-a e $5-b)$ :

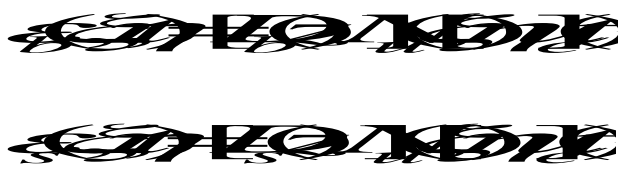

As energias, cinética e de deformação, na formulação em elementos finitos são expressas por: 


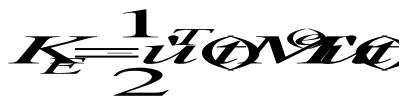

$$
\begin{aligned}
& s=\frac{1}{2} \text { का }
\end{aligned}
$$

Onde:

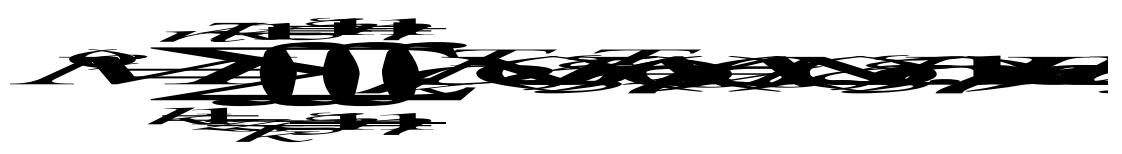

Onde $\mathrm{M}^{(\mathrm{e})}$ é a matriz de massa elementar, e $\mathrm{K}^{(\mathrm{e})}=\mathrm{K}_{\mathrm{b}}(\mathrm{e})+\mathrm{K}_{\mathrm{s}}(\mathrm{e})$ é a matriz de rigidez elementar, considerando os efeitos de flexão-membrana e de cisalhamento expressados por:

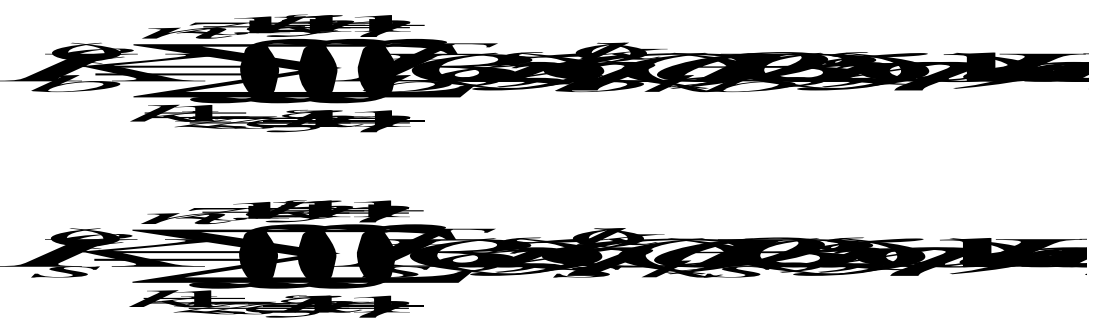

As matrizes $C_{b}{ }^{k}(\theta)$ e $C_{s}{ }^{k}(\theta)$ são ortotrópicas e estão em função do ângulo de orientação das fibras, ou seja, o material compósito está em função das coordenadas locais, assim é conveniente utilizar uma matriz de transformação, para promover a transformação do sistema de coordenadas locais do material para coordenadas globais.

A partir do cálculo das matrizes elementares podemos escrever a equação global do movimento no domínio do tempo da seguinte forma:

$$
\text { ICD }
$$

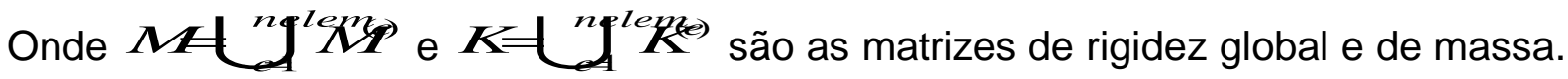
$\mathrm{O}$ símbolo $\mathrm{U}$ indica a matriz de montagem, $\mathrm{q}(\mathrm{t})$ é o vetor de deslocamento global $\mathrm{e}$ $\mathrm{f}(\mathrm{t})$ é o vetor de cargas generalizadas.

A Eq. (10) pode ser expressa pela conforme a equação a seguir e assim tem-se a matriz de rigidez dinâmica complexa no domínio da frequência:

$$
Z(\omega)=K_{e}+G(\omega) \bar{K}_{v}-\omega^{2} M
$$

\section{FORMULAÇÃO NUMÉRICA PELA TEORIA HSDT}

De modo similar para teoria HSDT tem-se que os deslocamentos em um ponto arbitrário do elemento são expressos da seguinte forma (Lo et. al [4]):

$$
\boldsymbol{U}(x, y, z, t)=\boldsymbol{A}(z) \boldsymbol{u}(x, y, t)
$$


$\mathrm{Na} \mathrm{Eq.} \mathrm{(12),} \mathrm{tem-se:}$

$$
\begin{aligned}
& \boldsymbol{U}(x, y, z, t)= {[u(x, y, z, t) v(x, y, z, t) w(x, y, z, t)]^{T} } \\
& \boldsymbol{A}(z)=\left[\begin{array}{cccccccccccc}
1 & 0 & 0 & z & 0 & 0 & z^{2} & 0 & 0 & z^{3} & 0 \\
0 & 1 & 0 & 0 & z & 0 & 0 & z^{2} & 0 & 0 & z^{3} \\
0 & 0 & 1 & 0 & 0 & z & 0 & 0 & z^{2} & 0 & 0
\end{array}\right] \\
& \boldsymbol{u}(x, y, t)= {\left[u_{0}(x, y, t) v_{0}(x, y, t) w_{0}(x, y, t) \psi_{x}(x, y, t) \psi_{y}(x, y, t) \psi_{z}(x, y, t) \ldots\right.} \\
&\left.\ldots \zeta_{x}(x, y, t) \zeta_{y}(x, y, t) \zeta_{z}(x, y, t) \Phi_{x}(x, y, t) \Phi_{y}(x, y, t)\right]^{T}
\end{aligned}
$$

Onde $u(x, y, z, t), \quad v(x, y, z, t), \quad \mathrm{e} \quad w(x, y, z, t)$ denotam, respectivamente, os deslocamentos nas direções $x, y$ e $z$. $\left(u_{0}, v_{0}, w_{0}\right)$ e $\left(\psi_{x}, \psi_{y}, \psi_{z}\right)$ são, respectivamente, os deslocamentos do plano médio e as rotações da seção transversal nas direções $x, y$ e $z$. Os termos $\zeta_{x}, \zeta_{y}, \zeta_{z}, \Phi_{x}$ e $\Phi_{y}$, podem ser vistos como rotações de alta ordem, não possuindo uma interpretação geométrica clara (Diacenco [5]). Da Eq. (12), pode ser visto que a aproximação do deslocamento na direção da espessura $z$ é feita separadamente em um procedimento similar ao de separação de variáveis.

As relações deslocamentos-deformações usuais são usadas e as deformações resultantes são separadas em deformações de flexão e de cisalhamento, $\boldsymbol{\varepsilon}_{b}$ e $\boldsymbol{\varepsilon}_{s}$, respectivamente, como segue:

$$
\begin{aligned}
& \boldsymbol{\varepsilon}_{b}(x, y, z, t)=\left[\boldsymbol{D}_{0}+z \boldsymbol{D}_{1}+z^{2} \boldsymbol{D}_{2}+z^{3} \boldsymbol{D}_{3}\right] \boldsymbol{u}(x, y, t)=\boldsymbol{D}_{\boldsymbol{b}}(z) \boldsymbol{u}(x, y, t) \\
& \boldsymbol{\varepsilon}_{s}(x, y, z, t)=\left[\boldsymbol{D}_{4}+z \boldsymbol{D}_{5}+z^{2} \boldsymbol{D}_{6}\right] \boldsymbol{u}(x, y, t)=\boldsymbol{D}_{s}(z) \boldsymbol{u}(x, y, t)
\end{aligned}
$$

Onde $\quad \varepsilon_{b}(x, y, z, t)=\left[\varepsilon_{x x} \varepsilon_{y y} \varepsilon_{z z} \gamma_{x y}\right]^{T} \quad$ e $\quad \varepsilon_{s}(x, y, z, t)=\left[\gamma_{y z} \gamma_{z x}\right]^{T} . \quad \varepsilon_{x x}=\partial u / \partial x$, $\varepsilon_{y y}=\partial v / \partial y, \quad \varepsilon_{z z}=\partial w / \partial z, \quad \gamma_{x y}=(\partial u / \partial y+\partial v / \partial x), \quad \gamma_{y z}=(\partial v / \partial z+\partial w / \partial y) \quad \mathrm{e}$ $\gamma_{z x}=(\partial u / \partial z+\partial w / \partial x)$. As matrizes $\boldsymbol{D}_{i}(i=0, \ldots, 6)$ são compostas por operadores diferenciais que aparecem nas relações deslocamentos-deformações, como detalhado por (de Lima et al. [6]).

\section{PROCEDIMENTO NUMÉRICO}

A aplicação numérica tem por objetivo analisar o comportamento do material compósito do tipo laminado. O material analisado possui cinco camadas com orientações diferentes, a saber: $45^{\circ} / 0^{\circ} / 45^{\circ} / 0^{\circ} / 45^{\circ}$ de dimensões $10 \mathrm{~cm}$ por $10 \mathrm{~cm}$.

As propriedades elásticas são: $\quad \bar{E}_{1}=172,4 G P a, \quad \bar{E}_{2}=\bar{E}_{3}=6,89 G P a$, $\bar{G}_{12}=\bar{G}_{13}=3,45 G P a, \quad \bar{G}_{23}=1,38 G P a, \quad v_{12}=v_{13}=0,25, \quad v_{23}=0,30, \quad \rho=1566 \mathrm{~kg} / \mathrm{m}^{3}$ (densidade do material).

A Figura 2 mostra a malha de elementos finitos. 


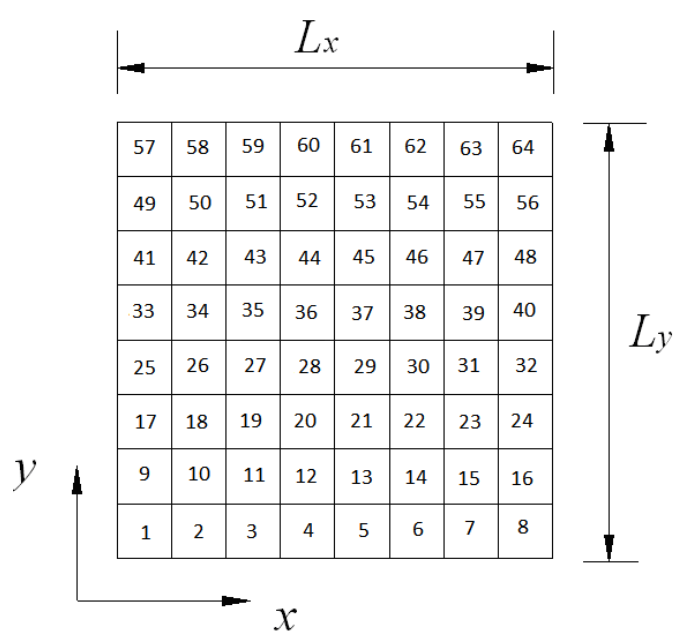

Figura 2 - Discretização da placa. Fonte: (Diacenco [7])

São consideradas as seguintes condições de contorno para o problema: $u 0=w 0=$ $\psi z=\varsigma x=\varsigma z=0$ em $y=0$ e $y=a$, e $u 0=w 0=\psi z=\varsigma y=\varsigma z=0$ em $x=0$ e $x=b$.

\subsection{APLICAÇÃO NUMÉRICA 1}

A Figura 3 ilustra as amplitudes da função de resposta em frequência (FRF) da estrutura composta laminada utilizando a Teoria FSDT (a) e a Teoria HSDT (b). Pode-se notar a presença de ruído nas FRFs. Foi aplicado um comando svd (singular value decomposition) do MatLab que consiste em um método para a fatoração de matrizes ortogonais, no caso do presente trabalho esta ferramenta foi aplicada para atuar como uma espécie de filtro para eliminar os ruídos que aparecem nas FRFs de ambas as teorias, os resultados são mostrados nas Figuras 4 (a) e (b).
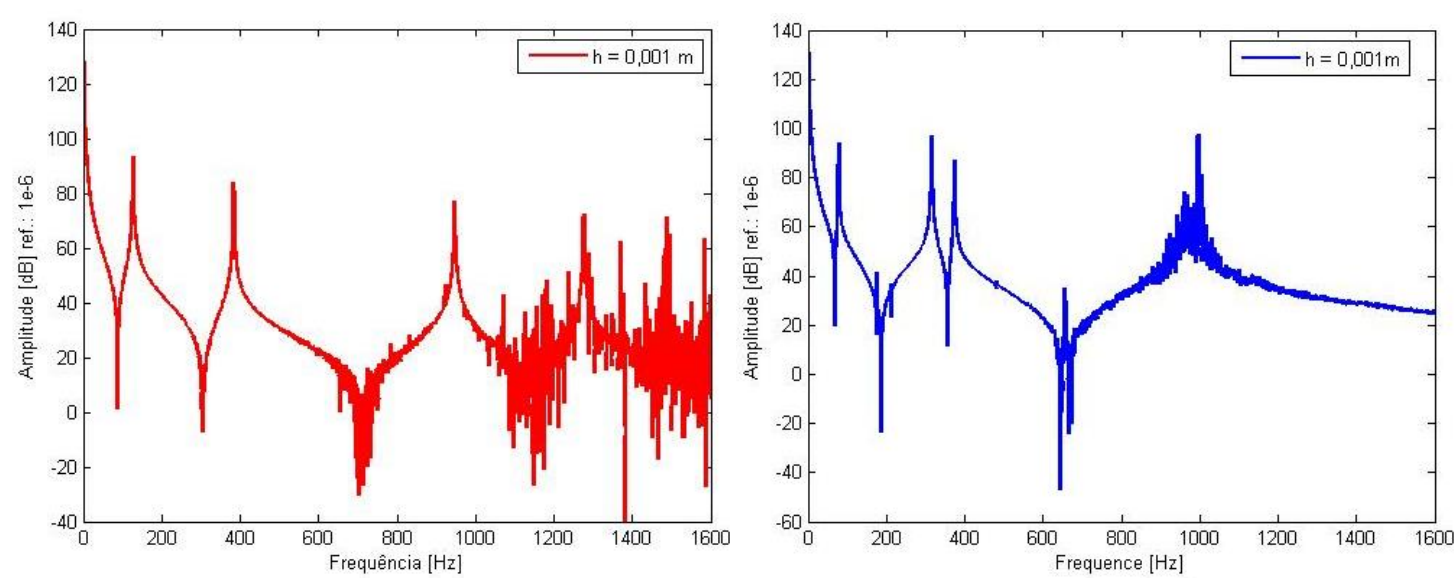

Figura 3: (a) FRFs sem a utilização de filtro obtido pela Teoria FSDT (b) FRFs sem a utilização de filtro obtido pela Teoria HSDT 

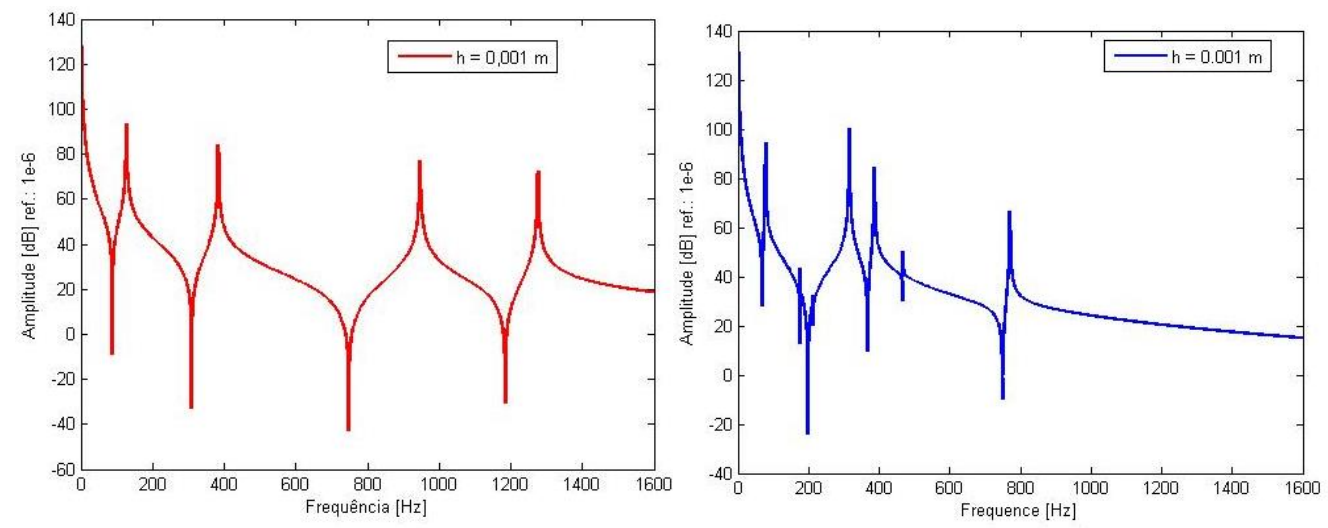

Figura 4: (a) FRFs com a utilização de filtro obtido pela Teoria FSDT (b) FRFs com a utilização de filtro obtido pela Teoria HSDT

\subsection{APLICAÇÃO NUMÉRICA 2}

Nesta aplicação compara-se o desempenho de ambas as teorias quando o parâmetro espessura das placas compósitas aumenta. Foi considerado que a espessura de todas as camadas de material compósito foi de $0,01 \mathrm{~m}$.

A Figura 5 ilustra as amplitudes da função de resposta em frequência (FRF) da estrutura composta laminada utilizando a Teoria FSDT (a) e a Teoria HSDT (b). Observa-se que, conforme a literatura apresenta, a capacidade de predição do comportamento dinâmico representado por meio das FRF's utilizando a Teoria FSDT fica comprometida.
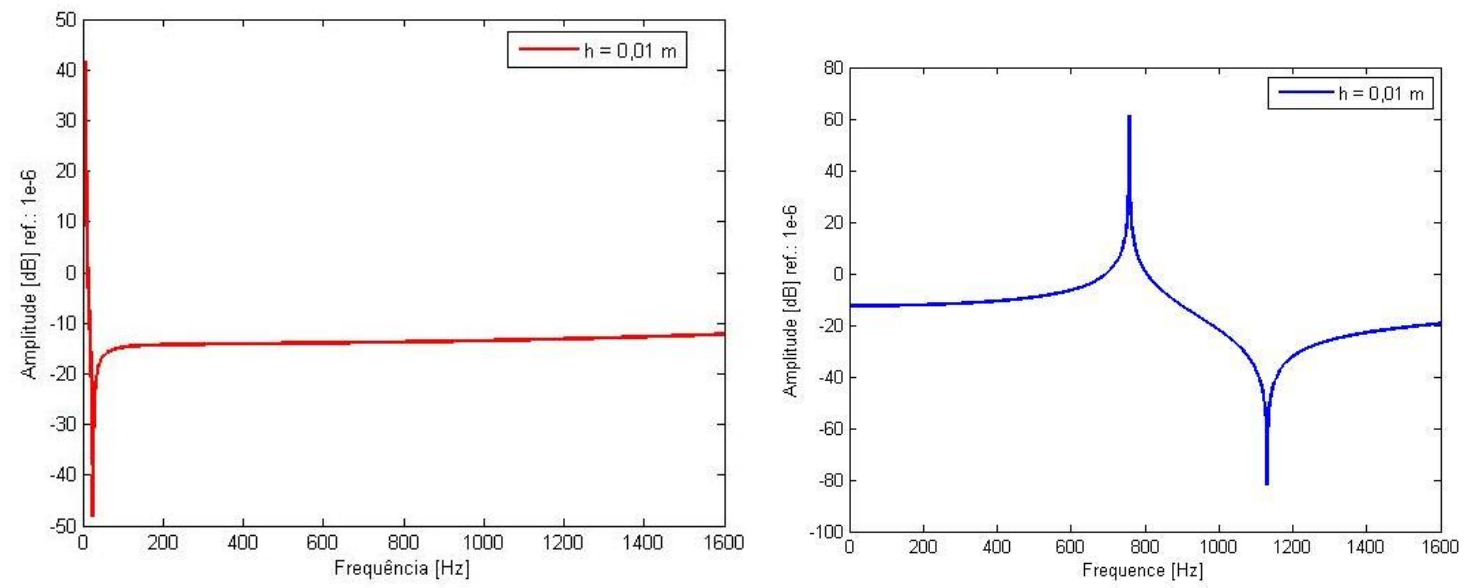

Figura 5: (a) FRF's com a utilização de filtro obtido pela Teoria FSDT e com o aumento da espessura das camadas compósitas (b) FRF's com a utilização de filtro obtido pela Teoria HSDT e com o aumento da espessura das camadas compósitas

\section{CONCLUSÕES}

Foi realizada uma análise comparativa entre duas teorias das que são mais utilizadas na modelagem de placas compósitas. Pode-se perceber que a Teoria FSDT apresenta limitações quando aumenta-se a espessura das camadas de material compósito, 
Foi realizado um estudo das técnicas de modelagem numérico-computacional de estruturas compostas laminadas incorporando tratamentos viscoelásticos superficiais, para fins de atenuação de vibrações. Em particular, foi utilizada a Teoria da Deformação de Ordem Superior a qual foi implementada em elemento Serendipity de placa retangular contendo oito nós e onze graus de liberdade por nó. Para representar o comportamento dinâmico do material viscoelástico em função da frequência de excitação e temperatura, foi utilizado o modelo do módulo complexo, o qual foi combinado com modelos de elementos finitos de sistemas estruturais laminados do tipo placas planas laminadas.

\section{AGRADECIMENTOS}

Os autores agradecem a FAPEMIG pelo apoio financeiro concedido a publicação deste artigo.

\section{REFERÊNCIAS}

1 Soriano, H. L. Método de Elementos Finitos em Análise de Estruturas. Editora USP, 1 Ed., São Paulo, SP, 2003. v. 1500. 604 p. Florida: CRC Press, 1997.

2 REDDY, J. N. Mechanics of Laminated Composite Plates: Theory and Analysis. 2.ed. Florida: CRC Press, 1997.

3 CEN, S., SOH, A., LONG, Y.; YAO, Z. A New 4-node Quadrilateral FE Model with Variable Electrical Degrees of Freedom for the Analysis of Piezoelectric Laminated Composite Plates. Composite Structures. v. 58, n.4, p. 583-599, 2002.

4 LO, K. H; CHRISTENSEN, R. M.; WU, E. M. A High Order Theory of Plate Deformation - Part 1: Homogeneous Plates. Journal of Applied Mechanics. v. 44, p. 663-668, 1977.

5 DIACENCO, A. A. Modelagem por Elementos Finitos de Materiais Compósitos Estruturais Incorporando Material Viscoelástico para o Controle Passivo de Vibração e Ruído. Dissertação De Mestrado, Universidade Federal de Itajubá, 2010.

6 de Lima, A.M.G., Ait Brik, B., Bouhaddi, N., Rade, D.A., 2006c, Multi-objective Optimization of Viscoelastically Damped Systems Combining Robust Condensation and Metamodels. Proceedings of the Eighth International Conference on Computational Structures Technology, Las Palmas de Gran Canaria, Spain.

7 DIACENCO, A. A. Modelagem de Otimização Aplicada à Topologia de Sensores para Identificação e Localização de Danos em Placa Compósita. 2016. Tese de Doutorado, Universidade Federal de Itajubá, MG. 